OPEN ACCESS

Edited by:

Maurizio Acampa,

Siena University Hospital, Italy

Reviewed by:

Silvia Schönenberger,

Heidelberg University

Hospital, Germany

Nicola Tarantino,

Montefiore Medical Center.

United States

*Correspondence:

Yueqiao Xu

xuyueqiao@sina.com

Specialty section:

This article was submitted to

Stroke,

a section of the journa

Frontiers in Neurology

Received: 10 December 2019

Accepted: 26 March 2020

Published: 28 April 2020

Citation:

Wang T, Xu Y, Wang N, Qi M, Cheng $W$ and $Q u X$ (2020) Effect of Remote Ischemic Conditioning in

Patients With Takotsubo Syndrome After Acute Stroke: Study Protocol for a Randomized Controlled Trial. Front. Neurol. 11:286. doi: 10.3389/fneur.2020.00286

\section{Effect of Remote Ischemic Conditioning in Patients With Takotsubo Syndrome After Acute Stroke: Study Protocol for a Randomized Controlled Trial}

\author{
Tao Wang, Yueqiao Xu* Ning Wang, Meng Qi, Weitao Cheng and Xin Qu \\ Department of Neurosurgery, Xuanwu Hospital, Capital Medical University, Beijing, China
}

Introduction: Takotsubo syndrome (TTS) is an acute heart failure syndrome which is preceded by a variety of emotional or physical triggers, with central nervous system conditions being an important trigger. Remote ischemic conditioning $(R \mid C)$ is a promising interventional treatment based on the probability that both TTS and acute coronary syndrome may respond similarly to interventions. The heart protection effect of RIC has been repeatedly confirmed in animal models and observational clinical trials; however, it has never been studied in patients with TTS after acute stroke in randomized clinical trials with a higher level of evidence. The present study will be a proof-of-concept study to determine whether RIC can reduce cardiac injury and eventually improve the heart function and clinical outcomes of TTS patients after acute stroke.

Methods and Analysis: A single-center, outcome-assessor-blinded, randomized controlled trial (RCT) will be conducted to evaluate the effect of RIC in TTS patients after acute stroke. Major eligibility criteria include TTS patients diagnosed with acute stroke, which can be confirmed on computed tomography or magnetic resonance imaging; patients aged 18-75 years; patients admitted to a hospital within $48 \mathrm{~h}$ after the onset of acute stroke; and patients diagnosed with Takotsubo cardiomyopathy with an InterTAK diagnostic score $\geq 50$. A total of 60 eligible patients will be randomly allocated into either the RIC or the control group. The primary endpoint is a composite of death from any cause and major adverse cardiac and cerebrovascular events during the in-hospital period and at the 1- and 6-month follow-up.

Ethics and dissemination: This study has been approved by the Medical Ethics Committee of Xuanwu Hospital, Capital Medical University ([2017] 072). The study findings will be presented at international conferences and published in a peer-reviewed journal.

Trial registration: This study has been prospectively registered in the Chinese Clinical Trial Registry on September 10, 2018 (ChiCTR1800018290).

Keywords: stroke, remote ischemic conditioning, Takotsubo syndrome, randomized controlled trial, study protocol 


\section{INTRODUCTION}

\section{Description of the Disease}

Takotsubo syndrome (TTS) represents an acute heart failure condition which is often preceded by sudden extreme stresses including emotional or physical triggers (1). A variety of physical stressors have been reported, including acute critical illness, acute respiratory failure, pancreatitis, traumatic injury, and iatrogenic factors such as surgery, exercise tests, diarrhea, or dobutamine stress echocardiography $(1,2)$. Central nervous system conditions such as stroke, traumatic brain injury, intracerebral hemorrhage, or seizures also represent an important trigger (1). Patients with TTS usually present with acute chest pain, breathlessness, palpitations, sweating, nausea, and congestive heart failure $(1,2)$ its clinical symptoms are similar to acute coronary syndrome, except demonstrable coronary artery stenosis or spasm (3). TTS is characterized by transient left ventricular dysfunction, which extends to more than one epicardial coronary artery territory, and a disproportionate increase in troponin compared with the diffuse electrocardiographic and cardiac imaging changes (4). Currently, there are no widely accepted diagnostic criteria for TTS. The most widely used criterium was the modified proposed criteria by the Mayo Clinic for the diagnosis of TTS (5). The InterTAK International Registry Group has developed a simple scoring system, the InterTAK diagnostic score (6), that takes into account five historically used clinical variables and two ECG variables. The InterTAK diagnostic score is slightly different from the modified Mayo Clinic criteria (7). Although considered as a fully reversible disease in most cases, TTS has been described in about $52 \%$ of cases to be associated with various complications, such as acute congestive heart failure, acute pulmonary edema, shock, and ventricular fibrillation, and it is in acute phase in most cases (8-11).

Several hypotheses have been proposed for the pathogenesis of TTS, including aborted myocardial infarction (MI) with spontaneous recanalization, myocarditis, coronary artery vasospasm, blood-borne catecholamine myocardial toxicity, and autonomic nervous system dysfunction with sympathetic hyperexcitation $(12,13)$, of which, the involvement of the adrenergic cascade triggered by physical or emotional stress is considered to be the most convincing $(14,15)$. Additionally, catecholamines can exert a direct catecholamine toxic effect on myocytes, involving the oxidative stress and inflammatory cascades (16), which can cause microvascular dysfunction, thus leading to reduced myocardial perfusion (17).

Abbreviations: TTS, Takotsubo syndrome; SAH, subarachnoid hemorrhage; ECG: electrocardiograph; MI: myocardial infarction; RIC: remote ischemic conditioning; RCT: randomized controlled trial; HS-CRP, high-sensitivity Creactive protein; IL-6, interleukin-6; IL-10, interleukin-10; IL-17, interleukin-17; LVEDD, left ventricular end-diastolic diameter; CRF, case record form; mRS, modified Rankin Scale; GOS, Glasgow Outcome Score; ChiCTR, Chinese Clinical Trial Registry; TRPV1, transient receptor potential vanilloid 1; CGRP, calcitonin gene-related peptide.

\section{Description of the Intervention}

Previous studies revealed that both neuronal and humoral components are involved in the signal transfer in remote ischemic conditioning (RIC) treatment; they act in concert and interact on three different levels from the stimulation site to the target organ. Firstly, peripheral sensory nerves are directly activated by the ischemic/reperfusion stimuli and/or indirectly activated by the humoral factors (18). Secondly, the peripheral sensory afferent nerves projecting to the autonomic centers of the central nervous system eventually activate efferent vagal nerves (18), which subsequently release acetylcholine to directly activate receptors in the target organs and/or activate receptors in other organs that can release humoral factors to indirectly act on the target organs (19). Finally, the intrinsic nervous system and the response of the specific parenchymal cells of the target organ are activated to contribute to the final effect $(19,20)$. Though transient receptor potential vanilloid 1 (TRPV1), calcitonin generelated peptide (CGRP), substance $\mathrm{P}$, and other mediators were found to be involved in RIC, the exact mechanisms of these pathways are still far from clear $(18,21-23)$. In this present study, a preliminary exploration on the changes in cardiac biomarkers will be made after RIC treatment; however, more basic research is still required to clarify the specific mechanism.

\section{Rationale for the Present Study}

Though the role of myocardial stunning in the mechanism of TTS is still unclear, some therapeutic algorithms have been developed for the treatment of TTS patients (1) based on the probability that both TTS and acute coronary syndrome may respond similarly to pharmacological or other interventions (24), among which, RIC is a promising intervention treatment. Ischemic conditioning was first recognized in 1986, when it was discovered that brief interruption of blood supply to a vascular territory limits the extent of ischemia, reperfusion injury, and the eventual size of the MI (25). Soon after, it was found that the brief and complete interruptions of blood supply to a particular vascular territory provide benefits to other vascular territories to a certain extent (26-29). Since then, the heart protection effect of RIC has been repeatedly confirmed in various animal models (30-36). Furthermore, several clinical studies have also shown the beneficial effects of RIC on heart injuries, such as ST-segment elevation myocardial infarction $(37,38)$, and heart failure after primary percutaneous coronary intervention (39). However, the heart protection effects of RIC have not been observed in all patients with heart injuries. Kahlert et al. reported that RIC provided no protection effect on the heart before transfemoral transcatheter aortic valve implantation in the interim analysis of a randomized controlled trial (RCT) (40). In particular, RIC has never been studied in patients with TTS patients after acute stroke. Therefore, whether RIC can benefit TTS patients after acute stroke still needs to be clarified.

\section{Objectives}

The present study will be a proof-of-concept study to determine whether RIC can exert a heart protection effect and thereby reduce cardiac injury and eventually improve the heart function and clinical outcomes of TTS patients after acute stroke. 


\section{METHODS AND ANALYSIS Overall Design}

A single-center, outcome-assessor-blinded, randomized controlled trial will be conducted in the Neurosurgical Intensive Care Unit (NICU) of Xuanwu Hospital, a tertiary neurosurgical referral centre in Beijing, China, and aims to evaluate the effect of RIC on TTS in patients with acute stroke. This protocol has been developed according to the Standard Protocol Items: Recommendations for Interventional
Trials (SPIRIT) (41). The conduction and reporting of this RCT will follow the Consolidated Standards of Reporting Trials (CONSORT) statement and its extension to nonpharmacological interventions (42). The study process is summarized in Figure 1.

\section{Eligibility Criteria}

Adult patients with acute stroke referred to the NICU will be screened for trial eligibility. Routine ECG, echocardiography,

\section{Enrollment}

Assessed for eligibility $(\mathrm{n}=\mathrm{N})$

- Diagnosed as acute stroke based on CT or MRI

- Hess-Hunt I-IV for patients with SAH

- Age between 18 and 75 years

- Within 48 hours after the onset of acute stroke

- Diagnosed as Takotsubo cardiomyopathy

- Provision of written informed consent

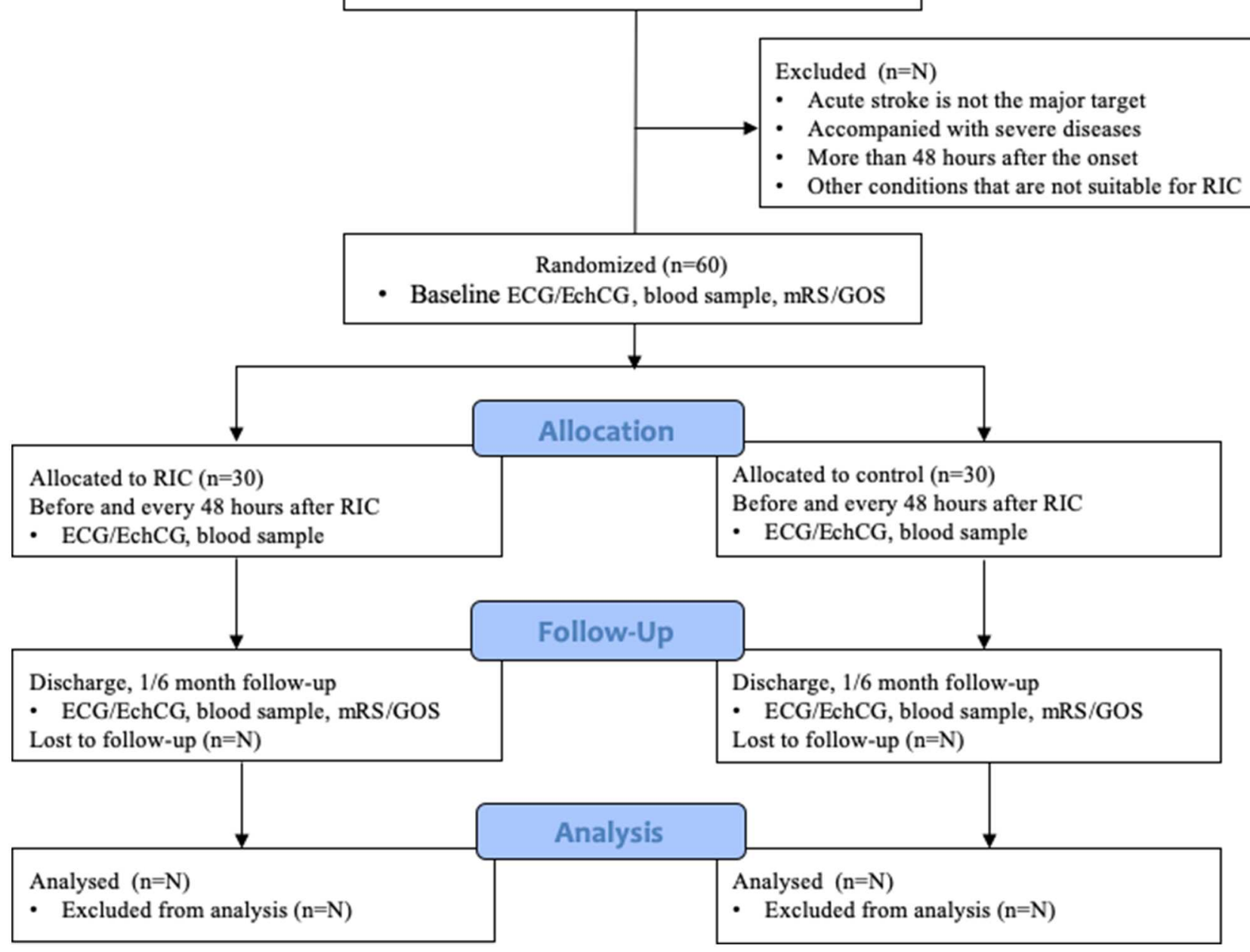

FIGURE 1 | Study flow diagram according to CONSORT 2010. SAH, subarachnoid hemorrhage; RIC, remote ischemic conditioning; ECG, electrocardiograph; EchCG, echocardiograph; mRS, modified Rankin Scale; GOS, Glasgow Outcome Score. 
and serum examination of cardiac injury biomarkers will be performed. Based on the InterTAK diagnostic score (6), patients diagnosed with TTS (InterTAK diagnostic score $\geq 50$ ) will be recruited into this study. At least two physicians will review the eligibility criteria, and a third senior physician will resolve any disagreement. The inclusion and exclusion criteria are summarized as follows:

\section{Inclusion Criteria}

1) Patients diagnosed with acute stroke (ischemic or haemorrhagic), which can be confirmed on computed tomography (CT) or MRI;

2) Patients diagnosed with subarachnoid hemorrhage with Hess-Hunt grades I-IV;

3) Patients aged 18-75 years;

4) Patients admitted to hospital within $48 \mathrm{~h}$ of acute stroke onset;

5) Patients diagnosed with Takotsubo syndrome with an InterTAK diagnostic score $\geq 50$ (6). The ventricular wall motion will be evaluated with echocardiography. If acute coronary syndrome (ACS) is suspected based on ECG, echocardiography, or biomarker findings, coronary computed tomography angiography (CTA) will be conducted to rule out ACS. Coronary angiography and cardiac magnetic resonance imaging will be utilized to differentiate ACS only when necessary.

6) Patients with a signed written informed consent form.

\section{Exclusion Criteria}

1) Acute stroke is not the major cause for admission or the patient is receiving palliative care.

2) The patients suffer from concurrent diseases, such as severe cardiovascular disease, are in an extremely critical condition, have cerebral hernia, and/or it is highly expected that the patients will fail to complete the study.

3) The patients are admitted to the hospital more than $48 \mathrm{~h}$ after acute stroke onset or have any subsequent stroke after the diagnosis of TTS.

4) The patients have concurrent limb vascular occlusive disease and/or other diseases or conditions which cause intolerance to the intervention treatment.

5) Both upper limbs of the patients are affected by the stroke, indicated by any symptoms, abnormal results of physical examination, or neurophysiologic tests in any upper limbs.

6) The patients have other conditions that are not suitable for RIC treatment, such as skin damage, severe thrombocytopenia, and abnormal blood clotting.

\section{Randomization and Blinding}

A total of 60 eligible patients with acute stroke, in accordance with the inclusion and exclusion criteria, will be included in this study. Random numbers will be generated on a computer by an unblinded physician who will not be involved in the data analysis and then placed in sealed envelopes. The patients will be randomly allocated into either the RIC or the control group according to a ratio of 1:1. The physician responsible for performing RIC will not be blinded and will play no further role in the clinical trial; the staff responsible for the data collection and analysis will be blinded. Hence, this is an outcome-assessorblinded study.

\section{Intervention and Follow-Up}

All eligible patients in the RIC and control groups will receive routine monitoring and medical treatment. Moreover, the patients in the RIC group will receive RIC treatment through an electric auto-control device that has been delineated by Ji et al. (patent number ZL200820123637.X, China): (43) a blood pressure cuff will be placed around the upper arm of the patient and inflated to a pressure of $200 \mathrm{mmHg}$ (44) for $5 \mathrm{~min}$ and then deflated for reperfusion for $5 \mathrm{~min}$. The inflation and deflation will be performed for four cycles each time, three times a day for a total of five consecutive days. If an upper limb is affected by the stroke, the other normal upper limb will be selected for RIC treatment. Blood samples will be collected before RIC treatment, every $48 \mathrm{~h}$ after RIC treatment until discharge from the NICU. The patients in the control group will not receive RIC treatment; however, blood samples will be collected at the same time points as those in the RIC group. Blood samples will be used for the detection of the following biomarkers: cardiac troponin I (cTnI), N-terminal pro B-type natriuretic peptide (NT-pro BNP), high-sensitivity C-reactive protein (HS-CRP), interleukin-6 (IL6), interleukin-10 (IL-10), interleukin-17 (IL-17), plasminogen activator inhibitor-1, plasma tissue plasminogen activator, microRNA-126, micro-RNA-155, serum nitric oxide (NO), blood uric acid, white blood cell count, and platelet aggregation rate. The electrocardiograph and echocardiography will be performed simultaneously every $48 \mathrm{~h}$ after RIC treatment until discharge from the NICU to evaluate the cardiac indicators such as ECG ST segment, percent of arrhythmia, ejection fraction, and left ventricular end-diastolic diameter (LVEDD). All eligible patients will be followed up by core team members based on clinical visits, medical records, or telephone interviews at 1 and 6 months after discharge and undergo the aforementioned examinations and serological tests (Table $\mathbf{1}$ ).

\section{Quality Control and Safety Assessment}

We will develop a manual describing in detail all aspects of the recruitment, eligibility criteria, randomization, interventions, data collection, and case record form (CRF) completion. All staff involved in this trial will be trained beforehand. This manual will be utilized to monitor adherence to procedural steps. Each cuff inflation/deflation time will be recorded to monitor adherence to the intended intervention. The study process will be supervised by an independent data monitoring committee. RIC is a noninvasive means to treat TTS patients by performing repeated inflation and deflation of a blood pressure cuff around the upper arm. However, it should be taken into account that there will be some possible adverse events, such as transient discomfort and/or ischemic damage of the upper arm during compression of the cuff, the formation of ecchymosis on the upper arm after RIC treatment, and new or aggravating neurological deficits during or after RIC treatment. Any serious adverse events possibly related to RIC treatment will be reported to the ethical committee. 
TABLE 1 | Data collection timeline.

\begin{tabular}{|c|c|c|c|c|}
\hline & $\begin{array}{l}\text { Cardiac examination } \\
\text { (electrocardiograph, } \\
\text { echocardiography) }\end{array}$ & Blood samples & $\begin{array}{l}\text { Patients' characteristics } \\
\text { (mRS or GOS) }\end{array}$ & Clinical outcomes \\
\hline At admission & $\checkmark$ & $\checkmark$ & $\checkmark$ & \\
\hline Before RIC & $\checkmark$ & $\checkmark$ & & \\
\hline Every $48 \mathrm{~h}$ after RIC & $\checkmark$ & $\checkmark$ & & \\
\hline At discharge from NICU & $\checkmark$ & $\checkmark$ & $\checkmark$ & $\checkmark$ \\
\hline At 1 month after discharge & $\checkmark$ & $\checkmark$ & $\checkmark$ & $\checkmark$ \\
\hline At 6 months after discharge & $\checkmark$ & $\checkmark$ & $\checkmark$ & $\checkmark$ \\
\hline
\end{tabular}

RIC, remote ischemic conditioning; NICU, neurosurgical intensive care unit; mRS, modified Rankin Scale; GOS, Glasgow Outcome Score.

\section{Patient and Public Involvement}

The protocol of this study was discussed and developed by a multidisciplinary team of experts including epidemiologists, clinicians, and statisticians; however, no patients or public people were involved in the design phase.

\section{Primary Endpoints}

The primary endpoint is a composite of death from any cause and major adverse cardiac and cerebrovascular events (a composite of a recurrence of Takotsubo syndrome, myocardial infarction, stroke or transient ischemic attack, or death from any cause) evaluated during the in-hospital period and at the 1- and 6month follow-up.

\section{Secondary Endpoints}

The secondary endpoints are death from any cause, a composite of major adverse cardiac and cerebrovascular events, serum cardiac biomarker cTnI, NT-pro BNP, modified Rankin Scale (mRS) score, Glasgow Outcome Score (GOS), cardiac function determined by echocardiography, ECG changes (QT interval and possible ST elevation), and blood uric acid level measured prior to RIC treatment, at discharge from the NICU, and at the 1- and 6-month follow-up. The average hospitalization duration will be recorded as a secondary endpoint to evaluate the efficiency of RIC.

\section{Sample Size}

No previous studies have focused on utilizing RIC to prevent TTS in patients after acute stroke. Most existing evidence utilized troponin I assay for the detection of cardiac injury. Power calculations for this proof-of-concept study will be based on serial troponin measurements, taken as continuous outcome variables with a predicted difference. Previous studies on RIC indicated a cardioprotective effect of RIC in reducing troponin levels by 18$62 \%(45-48)$. Based on the results of a pilot study in our center (the troponin level decreased by $40 \%, n=10$ ), we hypothesized a troponin level reduction of $37 \%$ with $80 \%$ power at the $5 \%$ significance level, and we calculated a sample size of 60 patients ( $n=30$ in each arm of the study).

\section{Data Management and Statistical Analysis}

An electronic database will be established to collect the data. Unique numeric identifiers will be assigned to each patient, which will be included in the study database; any other information related to the patient's identity will be excluded. Patients' identification will be solely used for follow-up purposes. Data safety will be supervised by an independent monitoring committee. Descriptive and summary statistics will be calculated for each treatment group for baseline characteristics. Continuous data will be expressed as means (standard deviations) or medians (interquartile ranges) for non-normal continuous data and ordinal data. Primary and secondary endpoints will be analyzed by the intent-to-treat principle. In univariate analyses, patientand clinical-related factors will be compared via the chi-square, analysis of variance, Student's $t$-test, or Mann-Whitney $U$ test, when appropriate, between two groups. The variables in the initial model included age, gender, diabetes status, hypertension, smoking status, serum cardiac biomarkers, blood uric acid, ejection fraction, LVEDD, mRS, and GOS at baseline. The relative prognostic significance of the variables in predicting the primary endpoint, mRS and GOS will be assessed by multivariate Cox regression models. Variables associated with a $p<0.10$ on univariable analysis were included in the multivariable analysis. Kaplan-Meier plots and log-rank tests will be utilized to compare the mRS and GOS between two groups. A $p<0.05$ will indicate statistical significance. SPSS 13.0 software (SPSS Inc., Chicago, IL) will be used for all statistical analyses.

\section{DISCUSSION}

We described the design and methods of a single-center, twoarm, parallel-group, randomized controlled trial investigating the efficacy of RIC in patients with TTS after acute stroke. This is the first RCT to address important questions about the heart protection effect of RIC in this population with such rigorous methodological design. It is expected that the study findings might hopefully become part of local protocols or international guidelines, allowing patients and care providers to make informed decisions and provide comprehensive information for future study designs. 
A limitation of this study is that it involves only one center and a minority of the subjects with TTS. This might influence the generalizability of the research results. Another limitation of this study is the non-blinding of the physician responsible for performing RIC. To reduce potential influence of this limitation on the outcomes, the physicians have no further role in the clinical trial and the staff responsible for data collection and analysis will be blinded.

\section{ETHICS AND DISSEMINATION}

This study has been approved by the Medical Ethics Committee of Xuanwu Hospital, Capital Medical University ([2017] 072), and the study findings will be presented at international conferences and published on a peer-reviewed journal. Safety considerations will be addressed as previously described.

\section{ETHICS STATEMENT}

This study protocol has confirmed with the declaration of Helsinki and has been reviewed and approved by the Medical Ethics Committee of Xuanwu Hospital, Capital Medical University ([2017] 072). All patients and/or their proxies will be asked to sign a written informed consent form.

\section{AUTHOR CONTRIBUTIONS}

YX was the principal investigator, has initiated the study, and applied for funding. TW was the study coordinator and has been involved in the study design and drafted this manuscript. NW,

\section{REFERENCES}

1. Ghadri JR, Wittstein IS, Prasad A, Sharkey S, Dote K, Akashi YJ, et al. International expert consensus document on takotsubo syndrome (part i): clinical characteristics, diagnostic criteria, and pathophysiology. Eur Heart J. (2018) 39:2032-46. doi: 10.1093/eurheartj/ehy076

2. Kato K, Lyon AR, Ghadri JR, Templin C. Takotsubo syndrome: aetiology, presentation and treatment. Heart. (2017) 103:14619. doi: 10.1136/heartjnl-2016-309783

3. Gopinath R, Ayya SS. Neurogenic stress cardiomyopathy: What do we need to know. Ann Cardiac Anaesthes. (2018) 21:228-34. doi: 10.4103/aca.ACA_176_17

4. Ranieri M, Finsterer J, Bedini G, Parati EA, Bersano A. Takotsubo syndrome: clinical features, pathogenesis, treatment, and relationship with cerebrovascular diseases. Curr Neurol Neurosci Rep. (2018) 18:20. doi: 10.1007/s11910-018-0833-7

5. Prasad A, Lerman A, Rihal CS. Apical ballooning syndrome (Tako-Tsubo or stress cardiomyopathy): a mimic of acute myocardial infarction. Ame Heart J. (2008) 155:408-17. doi: 10.1016/j.ahj.2007.11.008

6. Ghadri JR, Cammann VL, Jurisic S, Seifert B, Napp LC, Diekmann J, et al. A novel clinical score (InterTAK diagnostic score) to differentiate takotsubo syndrome from acute coronary syndrome: results from the International Takotsubo Registry. Eur J Heart Fail. (2017) 19:1036-42. doi: 10.1002/ejhf.683

7. Medina de Chazal H, Del Buono MG, Keyser-Marcus L, Ma L, Moeller FG, Berrocal D, et al. Stress cardiomyopathy diagnosis and treatment: JACC state-of-the-art review. J Am College Cardiol. (2018) 72:1955-71. doi: 10.1016/j.jacc.2018.07.072
MQ, WC, and XQ have been involved in the conception and study design. MQ has made important statistical contributions. All authors provided feedback on drafts of this paper and read and approved the final manuscript.

\section{FUNDING}

This trial will be supported by the Scientific Research Common Program of Beijing Municipal Commission of Education (grant no. KM201710025015), the Beijing Municipal 215 High-level Health Professional Personnel Academic Talent Project (grant no. 2015-3-062) and the National Natural Science Foundation of China (grant no. 81541120). The funders have no role in the design of the study, collection, analysis, interpretation of data, and preparation and publication of the manuscript.

\section{DISSEMINATION POLICY}

The researchers of this study have the intention to disseminate the results of the trial as widely as possible. Firstly, the trial has been registered on international clinical trial register database (registration number: ChiCTR1800018290, http://www. chictr.org.cn/showproj.aspx?proj=30637). Secondly, this study protocol will be published on a peer-reviewed journal. Finally, the findings will be presented at international meetings and disseminated to patients through peer-reviewed publications and newsletters. The identity of the patients will not be disclosed in any of these publication forms. The researchers of this study will attempt to publish the study results as soon as possible.
8. Elesber AA, Prasad A, Lennon RJ, Wright RS, Lerman A, Rihal CS. Four-year recurrence rate and prognosis of the apical ballooning syndrome. J Am College Cardiol. (2007) 50:448-52. doi: 10.1016/j.jacc.2007.03.050

9. Kurisu S, Inoue I, Kawagoe T. Conditions associated with left ventricular apical ballooning. Clin Cardiol. (2010) 33:E123-4. doi: 10.1002/clc.20726

10. Migliore F, Bilato C, Isabella G, Iliceto S, Tarantini G. Haemodynamic effects of acute intravenous metoprolol in apical ballooning syndrome with dynamic left ventricular outflow tract obstruction. Eur J Heart Failure. (2010) 12:305-8. doi: 10.1093/eurjhf/hfp205

11. Madhavan M, Rihal CS, Lerman A, Prasad A. Acute heart failure in apical ballooning syndrome (TakoTsubo/stress cardiomyopathy): clinical correlates and Mayo Clinic risk score. J Am College Cardiol. (2011) 57:1400-1. doi: 10.1016/j.jacc.2010.10.038

12. Y-Hassan S, Tornvall P. Epidemiology, pathogenesis, and management of takotsubo syndrome. Clin Autonomic Res. (2018) 28:53-65. doi: $10.1007 /$ s10286-017-0465-Z

13. Pelliccia F, Kaski JC, Crea F, Camici PG. Pathophysiology of takotsubo syndrome. Circulation. (2017) 135:2426-41. doi: 10.1161/circulationaha.116.027121

14. Lyon AR, Rees PS, Prasad S, Poole-Wilson PA, Harding SE. Stress (Takotsubo) cardiomyopathy-a novel pathophysiological hypothesis to explain catecholamine-induced acute myocardial stunning. Nat Clin Pract Cardiovasc Med. (2008) 5:22-9. doi: 10.1038/ncpcardio1066

15. Eitel I, Lücke C, Grothoff M, Sareban M, Schuler G, Thiele H, et al. Inflammation in takotsubo cardiomyopathy: insights from cardiovascular magnetic resonance imaging. Eur Radiol. (2010) 20:422-31. doi: $10.1007 / \mathrm{s} 00330-009-1549-5$ 
16. Galiuto L, De Caterina AR, Porfidia A, Paraggio L, Barchetta S, Locorotondo $\mathrm{G}$, et al. Reversible coronary microvascular dysfunction: a common pathogenetic mechanism in apical ballooning or tako-tsubo syndrome. Eur Heart J. (2010) 31:1319-27. doi: 10.1093/eurheartj/ehq039

17. Martin EA, Prasad A, Rihal CS, Lerman LO, Lerman A. Endothelial function and vascular response to mental stress are impaired in patients with apical ballooning syndrome. J Am College Cardiol. (2010) 56:1840-6. doi: 10.1016/j.jacc.2010.03.107

18. Gourine A, Gourine AV. Neural mechanisms of cardioprotection. Physiology. (2014) 29:133-40. doi: 10.1152/physiol.00037.2013

19. Basalay MV, Mastitskaya S, Mrochek A, Ackland GL, Del Arroyo AG, Sanchez J, et al. Glucagon-like peptide-1 (GLP-1) mediates cardioprotection by remote ischaemic conditioning. Cardiovasc Res. (2016) 112:669-76. doi: $10.1093 / \mathrm{cvr} / \mathrm{cvw} 216$

20. Pickard JM, Davidson SM, Hausenloy DJ, Yellon DM. Codependence of the neural and humoral pathways in the mechanism of remote ischemic conditioning. Basic Res Cardiol. (2016) 111:50. doi: 10.1007/s00395-016-0568-z

21. Heusch G. Remote ischemic conditioning: the enigmatic transfer of protection. Cardiovasc Res. (2017) 113:1-2. doi: 10.1093/cvr/cvw240

22. Heusch G, Botker HE, Przyklenk K, Redington A, Yellon D, et al. Remote ischemic conditioning. J Am College Cardiol. (2015) 65:177-95. doi: 10.1016/j.jacc.2014.10.031

23. Heusch G. Molecular basis of cardioprotection: signal transduction in ischemic pre-, post-, and remote conditioning. Circulat Res. (2015) 116:67499. doi: 10.1161/circresaha.116.305348

24. Madias JE. Remote ischemic postconditioning for patients with Takotsubo syndrome. Int J Cardiol. (2014) 172:e489-90. doi: 10.1016/j.ijcard.2014. 01.038

25. Murry CE, Jennings RB, Reimer KA. Preconditioning with ischemia: a delay of lethal cell injury in ischemic myocardium. Circulation. (1986) 74:1124-36.

26. Heusch G. Remote conditioning: the future of cardioprotection? J Cardiovasc Med. (2013) 14:176-9. doi: 10.2459/JCM.0b013e328358e507

27. Hausenloy DJ. Cardioprotection techniques: preconditioning, postconditioning and remote conditioning (basic science). Curr Pharm Des. (2013) 19:4544-63. doi: 10.2174/1381612811319250004

28. Birnbaum Y, Hale SL, Kloner RA. Ischemic preconditioning at a distance: reduction of myocardial infarct size by partial reduction of blood supply combined with rapid stimulation of the gastrocnemius muscle in the rabbit. Circulation. (1997) 96:1641-6.

29. Przyklenk K, Bauer B, Ovize M, Kloner RA, Whittaker P. Regional ischemic 'preconditioning' protects remote virgin myocardium from subsequent sustained coronary occlusion. Circulation. (1993) 87:893-9.

30. Zhang L, Guo H, Yuan F, Hong ZC, Tian YM, Zhang XJ, et al. Limb remote ischemia per-conditioning protects the heart against ischemia-reperfusion injury through the opioid system in rats. Can J Physiol Pharmacol. (2018) 96:68-75. doi: 10.1139/cjpp-2016-0585

31. Xu Y, Yu Q, Yang J, Yuan F, Zhong Y, Zhou Z, et al. Acute hemodynamic effects of remote ischemic preconditioning on coronary perfusion pressure and coronary collateral blood flow in coronary heart disease. Acta Cardiol Sinica. (2018) 34:299-306. doi: 10.6515/acs.201807_34(4)0.20180317a

32. Zheng Y, Xiao M, Li L, Li J, Reinhardt JD, Lu X. Remote physiological ischemic training promotes coronary angiogenesis via molecular and cellular mobilization after myocardial ischemia. Cardiovasc Therap. (2017) 35:e12257. doi: 10.1111/1755-5922.12257

33. Ma LL, Kong FJ, Guo JJ, Zhu JB, Shi HT, Li Y, et al. Hypercholesterolemia abrogates remote ischemic preconditioning-induced cardioprotection: role of reperfusion injury salvage kinase signals. Shock. (2017) 47:363-69. doi: $10.1097 /$ shk.0000000000000737

34. Kumas M, Altintas O, Karatas E, Kocyigit A. Protective effect of ischemic preconditioning on myocardium against remote tissue injury following transient focal cerebral ischemia in diabetic rats. Arqu Brasil Cardiol. (2017) 109:516-26. doi: 10.5935/abc.20170164

35. Singh A, Randhawa PK, Bali A, Singh N, Jaggi AS. Exploring the role of TRPV and CGRP in adenosine preconditioning and remote hind limb preconditioning-induced cardioprotection in rats. Cardiovasc Drugs Therap. (2017) 31:133-43. doi: 10.1007/s10557-017-6716-3
36. Randhawa PK, Jaggi AS. Investigating the involvement of TRPV1 ion channels in remote hind limb preconditioning-induced cardioprotection in rats. Naunyn-Schmiedeberg's Arch Pharmacol. (2017) 390:117-26. doi: 10.1007/s00210-016-1311-x

37. Schmidt MR, Rasmussen ME, Bøtker HE. Remote ischemic conditioning for patients with STEMI. J Cardiovasc Pharmacol Therap. (2017) 22:302-09. doi: $10.1177 / 1074248417702481$

38. McLeod SL, Iansavichene A, Cheskes S. Remote ischemic perconditioning to reduce reperfusion injury during acute ST-segment-elevation myocardial infarction: a systematic review and meta-analysis. J Am Heart Assoc. (2017) 6:e005522. doi: 10.1161/jaha.117.005522

39. Ladejobi A, Wayne M, Martin-Gill C, Guyette FX, Althouse AD, Sharbaugh $\mathrm{MS}$, et al. Association of remote ischemic peri-conditioning with reduced incidence of clinical heart failure after primary percutaneous coronary intervention. Cardiovasc Revascul Med. (2017) 18:105-09. doi: 10.1016/j.carrev.2016.12.004

40. Kahlert P, Hildebrandt HA, Patsalis PC, Al-Rashid F, Jánosi RA, Nensa F, et al. No protection of heart, kidneys and brain by remote ischemic preconditioning before transfemoral transcatheter aortic valve implantation: Interim-analysis of a randomized single-blinded, placebo-controlled, single-center trial. Int J Cardiol. (2017) 231:248-54. doi: 10.1016/j.ijcard.2016.12.005

41. Chan AW, Tetzlaff JM, Altman DG, Laupacis A, Gøtzsche PC, KrleŽa-Jerić K, et al. SPIRIT 2013 statement: defining standard protocol items for clinical trials. Ann Inter Med. (2013) 158:200-7. doi: 10.7326/0003-4819-158-3-201302050-00583

42. Schulz KF, Altman DG, Moher D. CONSORT 2010 statement: updated guidelines for reporting parallel group randomised trials. BMC Med. (2010) 8:18. doi: 10.1186/1741-7015-8-18

43. Meng R, Asmaro K, Meng L, Liu Y, Ma C, Xi C, et al. Upper limb ischemic preconditioning prevents recurrent stroke in intracranial arterial stenosis. Neurology. (2012) 79:1853-61. doi: 10.1212/WNL.0b013e3182 $71 \mathrm{f} 76 \mathrm{a}$

44. Mayor F, Bilgin-Freiert A, Connolly M, Katsnelson M, Dusick JR, Vespa P, et al. Effects of remote ischemic preconditioning on the coagulation profile of patients with aneurysmal subarachnoid hemorrhage: a case-control study. Neurosurgery. (2013) 73:808-15. doi: 10.1227/neu.0000000000000098

45. Candilio L, Malik A, Ariti C, Barnard M, Di Salvo C, Lawrence D, et al. Effect of remote ischaemic preconditioning on clinical outcomes in patients undergoing cardiac bypass surgery: a randomised controlled clinical trial. Heart. (2015) 101:185-92. doi: 10.1136/heartjnl-2014-306178

46. Thielmann M, Kottenberg E, Kleinbongard P, Wendt D, Gedik N, Pasa S, et al. Cardioprotective and prognostic effects of remote ischaemic preconditioning in patients undergoing coronary artery bypass surgery: a single-centre randomised, double-blind, controlled trial. Lancet. (2013) 382:597-604. doi: 10.1016/s0140-6736(13)61450-6

47. Hausenloy DJ, Candilio L, Laing C, Kunst G, Pepper J, Kolvekar S, et al. Effect of remote ischemic preconditioning on clinical outcomes in patients undergoing coronary artery bypass graft surgery (ERICCA): rationale and study design of a multi-centre randomized double-blinded controlled clinical trial. Clin Res Cardiol. (2012) 101:339-48. doi: 10.1007/s00392-0110397-x

48. Hoole SP, Heck PM, Sharples L, Khan SN, Duehmke R, Densem CG, et al. Cardiac remote ischemic preconditioning in coronary stenting (CRISP Stent) study: a prospective, randomized control trial. Circulation. (2009) 119:820-7. doi: 10.1161/circulationaha.108.809723

Conflict of Interest: The authors declare that the research was conducted in the absence of any commercial or financial relationships that could be construed as a potential conflict of interest.

Copyright (c) 2020 Wang, Xu, Wang, Qi, Cheng and Qu. This is an open-access article distributed under the terms of the Creative Commons Attribution License (CC $B Y)$. The use, distribution or reproduction in other forums is permitted, provided the original author(s) and the copyright owner(s) are credited and that the original publication in this journal is cited, in accordance with accepted academic practice. No use, distribution or reproduction is permitted which does not comply with these terms. 